\title{
Scale Exploiting Minimal Solvers for Relative Pose with Calibrated Cameras
}

\author{
Stephan Liwicki \\ stephan.liwicki@crl.toshiba.co.uk \\ Christopher Zach \\ christopher.zach@crl.toshiba.co.uk
}

Toshiba Research Europe Ltd.

Cambridge, United Kingdom

\begin{abstract}
We present efficient minimal solvers for the estimation of relative pose between two calibrated images. The proposed method exploits known scale ratios of feature matches which are intrinsically provided by scale invariant feature detectors with scale-space pyramids (e.g. SIFT). Since we are using scale, the number of required feature matches is reduced and consequently fewer inliers are needed. In our paper, we derive two solvers each related to one of the two possible minimal sets: Our $1+3$ point algorithm estimates relative pose from four feature matches with one known scale, while our $2+1$ point algorithm computes relative pose from three feature matches with two known scales. We embed the proposed methods into RANSAC with two inlier classes, and present an efficient RANSAC modification for less reliable scales. Finally, we analyze performance and robustness in synthetic data, and evaluate our RANSAC approach on real data in comparison to RANSAC based on the five point algorithm.
\end{abstract}

\section{Introduction}

Relative pose estimation has been studied for centuries, first in the photographic and later in the computer vision community. Nevertheless, due to the fundamental relevance to many state-of-the-art applications in structure from motion and simultaneous localization and mapping, research is ever evolving in recent years. The core challenge of relative pose is to estimate the motion between two camera poses, revealing $3 \mathrm{D}$ reconstructions of the environment as well as camera odometry.

Classic approaches study the location of point correspondences in multiple views and their relationship to pose recovery $[\mathbf{0}, \square, \square, \square, \square]$. In the case of unknown intrinsic calibration, the 8 point algorithm or 7 point algorithm is most frequently employed to recover the fundamental matrix $[\boldsymbol{Q}, \mathbb{\square}]$, and the 6 point algorithm is used for unknown focal length [ख] . With calibrated cameras the 5 point algorithm is most suitable [ㅁ]. In our work we focus on the later situation where intrinsic camera parameters are available.

Recent literature tries to reduce the number of needed point correspondences by introducing additional hardware requirements or restrictive assumptions on the environment. In [四] a 4 point algorithm is introduced which requires known relative rotation angles between views, which may be provided by an inertial measuring unit, resulting in faster and more robust estimation of relative pose. Similarly, [ㅁ] propose a 3 point algorithm for efficient 


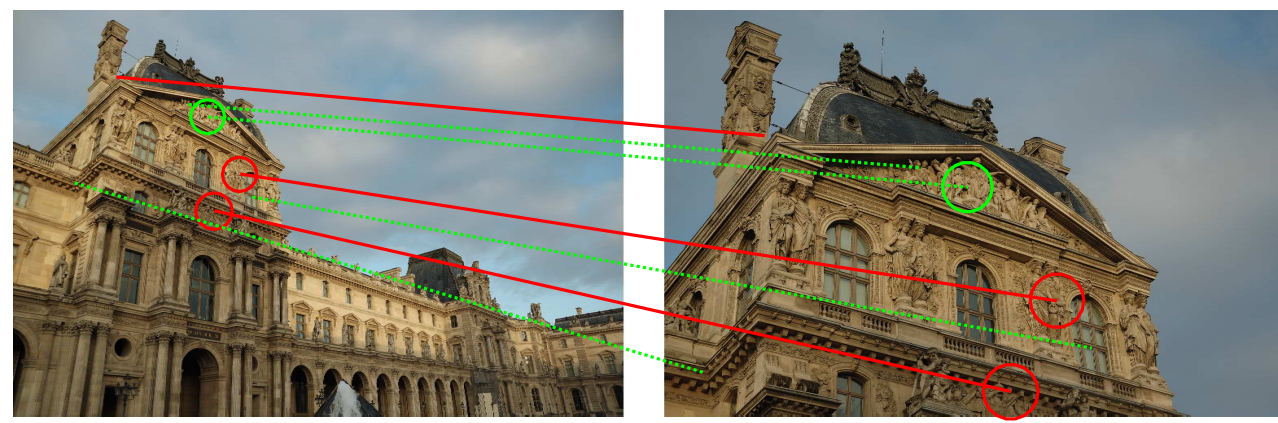

Figure 1: We utilize intrinsically known scale from feature matching with scale-space pyramids (e.g. SIFT) to compute relative pose between calibrated images. Two minimal solvers are introduced: one for 4 correspondences with 1 known scale (green), and one for 3 matching features with 2 scales (red).

visual odometry with known rotation orientations. Finally, $[\mathrm{\theta}]$ exploits the setting of vehicle motion to estimate relative pose for self-driving cars using the locations of 2 point correspondences alone. In contrast, we present two new minimal solvers that reduce the needed number of correspondences with minimal additional overhead.

\subsection{Contributions}

Inspired by feature matching strategies such as SIFT [ $\square$ ] or ORB [ㅁ] , which provide scale between correspondences, we introduce two solutions for relative pose with known scale ratios (fig. 1). In particular notice, as SIFT employs a scale-space pyramid, the patches' scales on the image planes are intrinsically provided. We exploit the connection between scales and depth and derive geometric constrains linking pose and depth ratios. In total, there are two possible minimal sets for location and scale correspondences: We derive our $1+3$ point algorithm which takes 4 feature matches with 1 known scale, and our $2+1$ point algorithm which estimates relative pose from 3 matches with 2 known scales.

Our final estimation framework employs random sampling consensus (RANSAC) which is a popular estimation strategy frequently employed in estimation tasks affected by outliers $[\square, \mathbb{\square}, \mathbf{\square}, \mathbf{\square}, \mathbb{G}]$. We adapt RANSAC to our setup, and present an efficient modification for unstable scales. Finally we analyse the performance and robustness of our systems in comparison to the 5 point algorithm in [ $\square]$. In summary our contributions are:

1. We derive geometric observations relating scale (or depth) ratios to relative pose.

2. We exploit scale-space feature detection and derive two new minimal solvers for pose.

3. We embed our $1+3$ and $2+1$ point algorithm into RANSAC for unreliable scale.

4. We analyse performance and robustness in synthetic and natural images.

In our work we use the reciprocal relation between scale and depth, leading to a similar setup to the stereo system in [వ]. In particular, $[\square]$ introduce a solver for pose between stereo cameras with minimal overlap. Due to the scale invariance of monocular images, their requirement of 4 points with 1 known depth value bares similarity to our $1+3$ point algorithm. Contrasting [ $[\mathbf{\nabla}$, we establish a $1+3$ and a $2+1$ point algorithm for monocular views, and introduce a methodology to exploit the scale-space of common feature detectors. 
Furthermore, we emphasize while $[\square]$ approximate the rotation matrix through linearization, we introduce an exact solver for the $1+3$ setup.

Finally we note, while our method extracts scales using areas surrounding feature points, its mechanism is in stark contrast to patch-based approaches in literature $[\square, \square, \square]$. Generally, these use affine patches or homographic frames from which additional point correspondences are created and applied to standard relative pose estimation techniques $[\mathbf{Q}, \mathbf{\square}, \mathbb{\square}, \mathbf{\square}]$. However, a drawback with this approach is that an inaccurate local frame leads to incorrect points and consequently to incorrect results. None of these works propose frame refinement. In contrast, our method solely relies on robustly estimated location and scale using efficient refinement methods.

\section{Geometric Observations}

We denote $N$ matched points in two views by homogenous vectors $\mathbf{x}_{i}=\left[\begin{array}{lll}x_{i} & y_{i} & 1\end{array}\right]^{\mathrm{T}}$ and $\mathbf{x}_{i}^{\prime}=\left[\begin{array}{ccc}x_{i}^{\prime} & y_{i}^{\prime} & 1\end{array}\right]^{\mathrm{T}}, i=1, \ldots, N$ respectively. Their corresponding 3D points in each frame's 3D coordinate system is found by $d_{i} \mathbf{x}_{i}$ or $d_{i}^{\prime} \mathbf{x}_{i}^{\prime}$, where $d_{i}$ and $d_{i}^{\prime}$ denote the depth of points as observed from the relevant view. Furthermore, let us express the relative pose between images by rotation $\mathbf{R} \in S O(3) \subset \mathbb{R}^{3 \times 3}$ and translation $\mathbf{t} \in \mathbb{R}^{3}$. Without loss of generality, we assume the intrinsic calibration matrix $\mathbf{K}$ to be the identity. Now the epipolar constraint is given by

$$
\left(\mathbf{x}_{i}^{\prime}\right)^{\mathrm{T}} \mathbf{E} \mathbf{x}_{i}=0 \text {, with essential matrix } \mathbf{E}=[\mathbf{t}]_{\times} \mathbf{R},
$$

where the cross-product between $\mathbf{t}$ and any vector $\mathbf{a} \in \mathbb{R}^{3}$ is expressed by $[\mathbf{t}]_{\times} \mathbf{a}=\mathbf{t} \times \mathbf{a}$.

Given ratio $s_{i}=\frac{d_{i}}{d_{i}^{\prime}}$ between depths $d_{i}$ and $d_{i}^{\prime}$ of correspondence $\left(\mathbf{x}_{i}, \mathbf{x}_{i}^{\prime}\right)$ from two views, we observe that the direction of the translation $\frac{\mathbf{t}}{|\mathbf{t}|}$ is determined by rotation $\mathbf{R}$, as

$$
\mathbf{t}=d^{\prime} \mathbf{x}^{\prime}-d \mathbf{R} \mathbf{x} \quad \Leftrightarrow \quad \mathbf{t}=d^{\prime}\left(\mathbf{x}^{\prime}-s \mathbf{R} \mathbf{x}\right) \quad \Rightarrow \quad \mathbf{t} \propto \mathbf{x}^{\prime}-s \mathbf{R} \mathbf{x} .
$$

Therefore, with one known correspondence $\left(\mathbf{x}, \mathbf{x}^{\prime}, s\right)$ we rewrite (1) as

$$
\left(\mathbf{x}_{i}^{\prime}\right)^{\mathrm{T}}\left(\mathbf{t} \times \mathbf{R} \mathbf{x}_{i}\right)=\left(\mathbf{x}_{i}^{\prime}\right)^{\mathrm{T}}\left(\left(\mathbf{x}^{\prime}-s \mathbf{R} \mathbf{x}\right) \times \mathbf{R} \mathbf{x}_{i}\right)=\left(\mathbf{x}_{i}^{\prime} \times \mathbf{x}^{\prime}\right)^{\mathrm{T}} \mathbf{R} \mathbf{x}_{i}-\left(\mathbf{x}_{i}^{\prime}\right)^{\mathrm{T}} \mathbf{R}\left(s \mathbf{x} \times \mathbf{x}_{i}\right)=0,
$$

using equalities $\mathbf{a}^{\mathrm{T}} \mathbf{b}=\mathbf{b}^{\mathrm{T}} \mathbf{a}, \mathbf{a}^{\mathrm{T}}(\mathbf{b} \times \mathbf{c})=\mathbf{b}^{\mathrm{T}}(\mathbf{c} \times \mathbf{a})$ and $\mathbf{R}(\mathbf{a} \times \mathbf{b})=\mathbf{R a} \times \mathbf{R b}$ for $\mathbf{a}, \mathbf{b}, \mathbf{c} \in \mathbb{R}^{3}$. Notice, rewriting (3) as the equality $\left(\mathbf{x}^{\prime}-s \mathbf{R} \mathbf{x}\right)^{\mathrm{T}}\left(\mathbf{R} \mathbf{x}_{i} \times \mathbf{x}_{i}^{\prime}\right)=0$ reveals the intuition behind: all epipolar planes must contain the translation $\mathbf{t}$ or equivalently the vector $\left(\mathbf{x}^{\prime}-s \mathbf{R} \mathbf{x}\right)$.

Furthermore, (3) entails that all correspondences with depth ratios $\left(\mathbf{x}_{i}, \mathbf{x}_{i}^{\prime}, s_{i}\right)$ satisfy

$$
\mathbf{t} \times\left(\mathbf{x}_{i}^{\prime}-s_{i} \mathbf{R} \mathbf{x}_{i}\right)=\mathbf{0} \quad \Leftrightarrow \quad \mathbf{t} \times \mathbf{x}_{i}^{\prime}-\mathbf{t} \times s_{i} \mathbf{R} \mathbf{x}_{i}=\mathbf{0} \quad \Leftrightarrow \quad \mathbf{t} \times \mathbf{x}_{i}^{\prime}=s_{i} \mathbf{t} \times \mathbf{R} \mathbf{x}_{i},
$$

where $\mathbf{a} \times \mathbf{b}=\mathbf{0} \Leftrightarrow \mathbf{a} \propto \mathbf{b}$ for $\mathbf{a}, \mathbf{b} \in \mathbb{R}^{3}$. Notice, (4) not only enforces the epipolar constraint though (3), it also ensures the sine rule of triangles holds. We employ (2), (3) and (4) to propose our minimal solvers in the following sections.

\section{Proposed Minimal Solvers}

We describe our approach for relative pose estimation between two calibrated monocular views. In contrast to many existing methods which use location alone, e.g. [Q, $\mathbf{\square}, \mathbf{\square}]$, we 
exploit known scale ratios of feature matches. Our motivation comes from commonly employed feature detectors and descriptors, which rely on scale-space pyramid matching, e.g. SIFT[प], ORB[ㅁ] . Specifically, these methods intrinsically provide the scale difference between matched features in addition to the location information. We notice, known scale ratio on the image plane is closely linked to the depth ratio. In fact, the scale ratio is reciprocal to the depth ratio in homogeneous coordinate space:

$$
\left[\begin{array}{l}
\frac{x}{s} \\
\frac{y}{s} \\
1
\end{array}\right] \doteq\left[\begin{array}{l}
x \\
y \\
s
\end{array}\right]
$$

We denote this ratio between scales, or equivalently depth values, as $s_{i}=\frac{d_{i}}{d_{i}^{\prime}}$ in the following

There are exactly two minimal sets which solve the relative pose problem under consideration of scale. In total we have 5 unknowns representing the transition between cameras, i.e. 3 for rotation and 3 for translation minus 1 as scale is irrecoverable. The $2 \mathrm{D}$ location of matching pixels provides one epipolar constraint. If scale is additionally provided, the sine rule in (4) must also hold. Henceforth there are two possible minimal solvers: (a) One point with scale and three points without $(1 \times 2+3 \times 1=5)$, and (b) two points with scale and one point without $(2 \times 2+1 \times 1=5)$. We introduce both in the subsequent sections.

\section{1 $1+3$ Point Algorithm}

Given a total of four matched points in the two image views, one with scale and location $\left(\mathbf{x}_{1}, \mathbf{x}_{1}^{\prime}, s_{1}\right)$ and three with location alone $\left(\mathbf{x}_{i}, \mathbf{x}_{i}^{\prime}\right), i=2, \ldots, 4$, we want to find rotation $\mathbf{R}$ and translation $\mathbf{t}$. Using (2) and (4), it is possible to express the following system of equations

$$
\begin{aligned}
\mathbf{x}_{1}^{\prime}-s_{1} \mathbf{R} \mathbf{x}_{1} & =\mathbf{t} \\
\left(\mathbf{x}_{i}^{\prime} \times \mathbf{x}_{1}^{\prime}\right)^{\mathrm{T}} \mathbf{R} \mathbf{x}_{i}-\left(\mathbf{x}_{i}^{\prime}\right)^{\mathrm{T}} \mathbf{R}\left(s_{1} \mathbf{x}_{1} \times \mathbf{x}_{i}\right) & =0, \quad i=2,3,4 .
\end{aligned}
$$

We note however, (7) is independent of $\mathbf{t}$, and hence forms a system of 3 equations with 3 unknowns for rotation $\mathbf{R}$. We use this part as the core of our minimal solver, coined $1+3$ point algorithm. Translation $\mathbf{t}$ is then found through (6), as length of $\mathbf{t}$ is irrecoverable.

We express rotation $\mathbf{R}$ as quaternion $q=q_{a}+q_{b} \mathbf{i}+q_{c} \mathbf{j}+q_{d} \mathbf{k}$, where

$$
\mathbf{R}=2\left[\begin{array}{ccc}
0.5-q_{c} q_{c}-q_{d} q_{d} & q_{b} q_{c}-q_{d} q_{a} & q_{b} q_{d}+q_{c} q_{a} \\
q_{b} q_{c}+q_{d} q_{a} & 0.5-q_{b} q_{b}-q_{d} q_{d} & q_{c} q_{d}-q_{b} q_{a} \\
q_{b} q_{d}-q_{c} q_{a} & q_{c} q_{d}+q_{b} q_{a} & 0.5-q_{b} q_{b}-q_{c} q_{c}
\end{array}\right] .
$$

Eq. (7) can now be brought into a simple quadratic form of 3 equations (see supplement)

$$
\mathbf{r}^{\mathrm{T}} \mathbf{A}_{i} \mathbf{r}+\mathbf{b}_{i} \mathbf{r}+c_{i}=0, \quad i=2,3,4
$$

where the vector of unknowns corresponds to $\mathbf{r}=\left[\begin{array}{ccc}\frac{q_{b}}{q_{a}} & \frac{q_{c}}{q_{a}} & \frac{q_{d}}{q_{a}}\end{array}\right]^{\mathrm{T}}$, and $\mathbf{A}_{i}, \mathbf{b}_{i}$ and $c_{i}$ are known observations. With rotation as homogenous quaternion, we derive a minimal solver through the Gröbner basis algorithm in [甘]. Our system resolves into an 8 degree univariate polynomial with 8 solutions. Its roots can be found efficiently through Sturm sequencing [四] or more reliably through eigenvalue decomposition [ $[\mathbf{6}, \mathbf{\square}]$ ].

Finally we emphasise, although (7) suffices as minimal solution, its naïve application to [ब] leads to a $60 \%$ increase in non-zero monomials and steps needed to form the Gröbner basis. Consequently, our modified set of equations produces a numerically more stable solver, as fewer computational steps are necessary. 


\section{2 $2+1$ Point Algorithm}

Let us now consider the minimal set with three point correspondences of which two have known scale, i.e. $\left(\mathbf{x}_{1}, \mathbf{x}_{1}^{\prime}, s_{1}\right),\left(\mathbf{x}_{2}, \mathbf{x}_{2}^{\prime}, s_{2}\right)$ and $\left(\mathbf{x}_{3}, \mathbf{x}_{3}^{\prime}\right)$. Using (2), (3) and (4), we start with the following system of equations

$$
\begin{aligned}
\mathbf{x}_{1}^{\prime}-s_{1} \mathbf{R} \mathbf{x}_{1} & =\mathbf{t} \\
\left(\mathbf{x}_{1}^{\prime}-s_{1} \mathbf{R} \mathbf{x}_{1}\right) \times\left(\mathbf{x}_{2}^{\prime}-s_{2} \mathbf{R} \mathbf{x}_{2}\right) & =\mathbf{0} \\
\left(\mathbf{x}_{3}^{\prime} \times \mathbf{x}_{1}^{\prime}\right)^{\mathrm{T}} \mathbf{R} \mathbf{x}_{3}-\left(\mathbf{x}_{3}^{\prime}\right)^{\mathrm{T}} \mathbf{R}\left(s_{1} \mathbf{x}_{1} \times \mathbf{x}_{3}\right) & =0,
\end{aligned}
$$

where (11) combines (4) with the proportional translation equivalence in (2). Similar to our $1+3$ point algorithm, a system to solve for rotation $\mathbf{R}$ alone is given by (11) and (12), which consists of three independent equations. We simplify (11), by first rearranging it into

$$
\mathbf{x}_{1}^{\prime} \times \mathbf{x}_{2}^{\prime}-s_{1} \mathbf{R} \mathbf{x}_{1} \times \mathbf{x}_{2}^{\prime}-s_{2} \mathbf{x}_{1}^{\prime} \times \mathbf{R} \mathbf{x}_{2}+s_{1} s_{2} \mathbf{R}\left(\mathbf{x}_{1} \times \mathbf{x}_{2}\right)=\mathbf{0}
$$

and then constructing a set of three equivalent quadratic equations, analogous to (9). When solving with Gröbner basis [], we obtain a 4 degree univariate polynomial with 4 solutions.

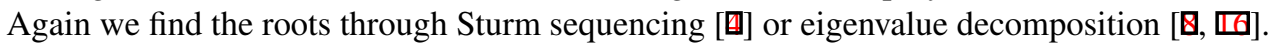

\subsection{Solutions and Degeneracies}

In contrast to methods which find the essential matrix, our methods computes rotation $\mathbf{R}$ and translation $\mathbf{t}$ directly. Therefore, chirality is automatically enforced. However, as with other methods, our $1+3$ and $2+1$ point algorithm has degeneracies. If points lie on the same epipolar plane, the equations are not independent resulting in a degeneracy. Also, as we express rotation as homogeneous quaternion, camera motion with rotation angle $180^{\circ}$ is unsupported. Both instances however are seldom experienced in real images.

\section{Random Sampling Consensus Integration}

RANSAC is a robust estimation framework which employs minimal solvers [ロ, $\square, \square, \square]$ ]. In particular, minimal sets are randomly selected to find hypotheses which are then tested on the remaining data samples. Here, we first consider the two class setup, and then unstable scales to integrate our minimal solvers into the RANSAC framework.

\subsection{Two Class RANSAC with Scale and Location}

Our minimal solvers have two classes of sample points, specifically locations of point correspondences with scale and without. Therefore, similarly to [వ], we modify the classical stopping criterion of RANSAC to reflect the two inlier types

$$
K=\frac{\log (1-p)}{\log \left(1-r_{s} r_{l}^{3}\right)} \quad \text { or } \quad K=\frac{\log (1-p)}{\log \left(1-r_{s}^{2} r_{l}\right)}
$$

for the $1+3$ and $2+1$ point algorithm respectively. $K$ is the number of iterations needed to guarantee an inlier set has been selected, up to a probability $p$, under the assumption that $r_{l}$ and $r_{s}$ represents the likelihood of selecting inliers for location and location with scale 


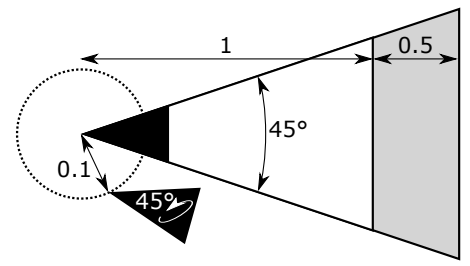

Figure 2: The cameras are chosen with focal length 1 and random motion with baseline 0.1 and rotation of at most $45^{\circ}$. Points are sampled with depth values between 1 and 1.5.

respectively. Notice, in comparison to the 5 point algorithm, if $r_{s}<r_{l}^{2}$ fewer iterations are needed. The rates are based on inlier tests. Commonly, the Sampson distance is employed for estimating the error of the epipolar constraint for testing locations [D]:

$$
\frac{\left(\left(\mathbf{x}^{\prime}\right)^{\mathrm{T}} \mathbf{K}_{2}^{-\mathrm{T}} \mathbf{E} \mathbf{K}_{1}^{-1} \mathbf{x}\right)^{2}}{\left(\mathbf{K}_{2}^{-\mathrm{T}} \mathbf{E} \mathbf{K}_{1}^{-1} \mathbf{x}\right)_{1}^{2}+\left(\mathbf{K}_{2}^{-\mathrm{T}} \mathbf{E} \mathbf{K}_{1}^{-1} \mathbf{x}\right)_{2}^{2}+\left(\mathbf{K}_{1}^{-\mathrm{T}} \mathbf{E}^{\mathrm{T}} \mathbf{K}_{2}^{-1} \mathbf{x}^{\prime}\right)_{1}^{2}+\left(\mathbf{K}_{1}^{-\mathrm{T}} \mathbf{E}^{\mathrm{T}} \mathbf{K}_{2}^{-1} \mathbf{x}^{\prime}\right)_{2}^{2}}<\delta_{l},
$$

where $\mathbf{K}_{1}$ and $\mathbf{K}_{2}$ are calibration matrices of the first and second view respectively, $(\mathbf{a})_{i}^{2}$ computes the square of the $i^{t h}$ element of vector $\mathbf{a}$, and $\delta_{l}$ is a location inlier threshold.

We now establish an inlier test for scale. With (4), and under the assumption that $\mathbf{x}$ and $\mathbf{x}^{\prime}$ are on the epipolar plane, we write

$$
\left|\mathbf{t} \times \mathbf{x}^{\prime}\right|=s|\mathbf{t} \times \mathbf{R x}| \quad \Leftrightarrow \quad \frac{\left|\mathbf{t} \times \mathbf{x}^{\prime}\right|}{|\mathbf{t} \times \mathbf{R x}|}=s \quad \text { and inlier test } \quad\left|\frac{\left|\mathbf{t} \times \mathbf{x}^{\prime}\right|}{|\mathbf{t} \times \mathbf{R x}|} \frac{1}{s}-1\right|<\delta_{s}
$$

as the normal vector of the cross-product is shared. The final term in (16) stems from the observation that scale from the image domain may differ from the expected scale implied by $\mathbf{R}$ and $\mathbf{t}$ which we denote $\hat{s}$ in the following. In particular, we define the scale error $\varepsilon_{s}$ as the change required to match $s$ with $\hat{s}$, such that the difference is given by $\hat{s}=s\left(1+\varepsilon_{s}\right)$ leading to our inlier test above. Finally, we define as scale inliers the correspondences which satisfy the location constraint in (15) as well as the scale test in (16) up to small errors $\delta_{l}$ and $\delta_{s}$. Notice, the magnitude of threshold $\delta_{s}$ can be easily established under consideration of parameters related to the step-size in the scale-space pyramid.

\subsection{Efficient Bisection Algorithm for Unstable Scale}

In high resolution images, feature detectors are fairly reliable for locations. Unfortunately, the estimation of scale is less stable and depends on the step-size used in the scale-space pyramid. For example, in SIFT scales are initially discretized by $s_{i} \in\left\{\sigma_{0} 2^{l \sigma_{s}} \mid l=0,1,2,3, \ldots\right\}$ with base scale $\sigma_{0}=1.6$ and step-size $\sigma_{s}=\frac{1}{3}$, and then interpolated to continuous values [四]. Consequently, errors are inadvertently introduced which can affect our solvers.

We propose an efficient bisection search which is aimed at correcting errors in scale. In particular, we solve the relative pose problem iteratively with multiple scales taken around the original observation. Starting with solutions for $s_{i}, s_{i}(1+\varepsilon)$ and $s_{i}(1-\varepsilon)$, we update the scale with the best choice for $s_{i}$, half the variance $\varepsilon \leftarrow \frac{\varepsilon}{2}$, and continue the process. Let us emphasize, a monotonous performance measure with reducing error is required for bisections to work. We employ the location inlier ratio as performance measure, which we empirically show to be valid in our experiments in section 5.1.2. 

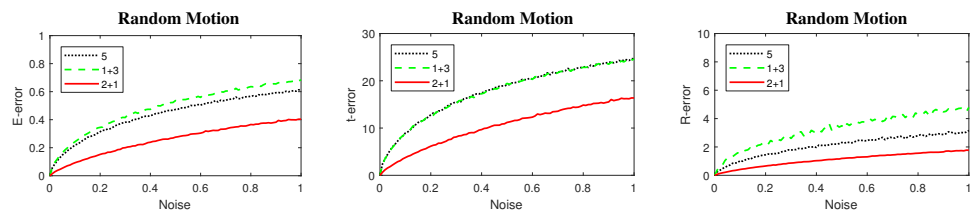

Figure 3: Estimation with noisy locations (noise is standard deviation in CIF image pixel) for random motion. Mean E-error, t-error and R-error of 10,000 instantiations for our $1+3$ and $2+1$ point algorithms are compared to the 5 point algorithm.
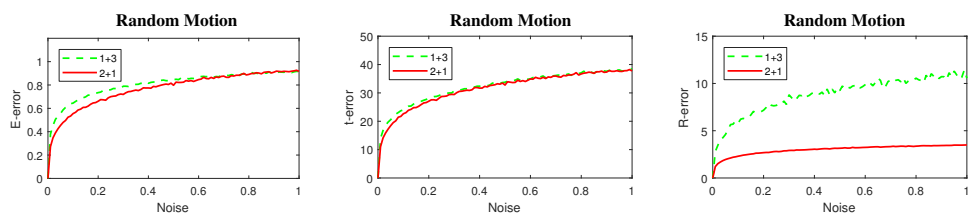

Figure 4: Estimation with noisy scales (Gamma distributed around 1 with variance of one $100^{\text {th }}$ of SIFT's step-size) for random motion. Mean E-error, R-error and t-error of the $1+3$ and $2+1$ point algorithms are shown. (5 point algorithm is uneffected and hence not shown.)

Finally we note, as we employ bisection search, more solutions are required to be computed for the relative pose problem. The scoring of the varied scales is computationally the most significant cost, as in contrast our solvers are fast. Hence, preemptive methods, e.g. [], and early bailout [] should be employed for enhanced runtime performance.

\section{Analysis and Experiments}

We analyse robustness and accuracy of our proposed minimal solvers with synthetic data, and with real images as part of a RANSAC setup.

\subsection{Performance Analysis in Noisy Conditions}

In this section we employ a synthetic data model to evaluate our methods with known noise. In particular, we follow [ $[\mathbf{0}]$ to generate a challenging two view setup (fig. 2). Additionally we introduce noise on location and depth ratio observations in the following.

Our evaluation is based on three error measures:

$$
\begin{array}{ll}
\text { E-error: } & \min _{i}\left\|\frac{\hat{\mathbf{E}}}{|\hat{\mathbf{E}}|}-\frac{\mathbf{E}_{i}}{\left|\mathbf{E}_{i}\right|}\right\|_{F} \\
\text { t-error: } & \min _{i} \arccos \left(\frac{\hat{\mathbf{t}}^{\mathrm{T}} \mathbf{t}_{i}}{|\hat{\mathbf{t}}|\left|\mathbf{t}_{i}\right|}\right) \\
\text { R-error: } & \min _{i} \arccos \left(\frac{\operatorname{trace}\left(\hat{\mathbf{R}}^{\mathrm{T}} \mathbf{R}_{i}\right)-1}{2}\right)
\end{array}
$$

where $i$ indexes the set of solutions. The Frobenius norm between the true essential matrix $\hat{\mathbf{E}}$ and estimated essential matrices $\mathbf{E}_{i}$ in (17) is used to test overall system performance. Angular errors between true translation vector $\hat{\mathbf{t}}$ and estimations $\mathbf{t}_{i}$ or true rotation matrix $\hat{\mathbf{R}}$ and estimations $\mathbf{R}_{i}$, in (18) and (19) respectively, are employed for a more detailed analysis. 

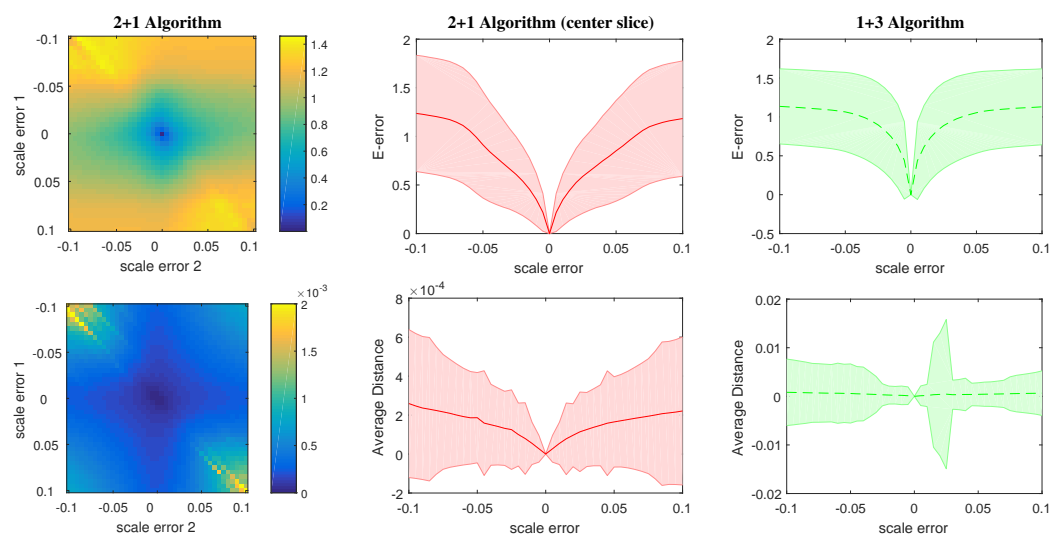

Figure 5: Scale effect on E-error (1st row) and the mean error on epipolar constraint for 10,000 inliers ( 2 nd row) is shown for 10,000 trials with $2+1$ (left, middle) and $1+3$ point algorithm (right). The slice at center of $2+1$ is shown with standard deviation, for comparison with $1+3$. Both methods increase virtually monotonically with increasing noise.

\subsubsection{Noise in Pixel Location}

Fig. 3 shows our obtained errors with noise in location measurements. Here we induce normal distributed noise to the points on the image planes, which we denote as a standard deviation of pixel magnitude as observed by a CIF image (i.e. resolution $352 p x \times 240 p x$ ). Our $1+3$ and $2+1$ point algorithms (denoted $1+3 \mathrm{pt}$ and $2+1 \mathrm{pt}$ in the following) are compared to the five point algorithm in [미 which considers location alone (denoted 5pt). We modify E-error for $5 \mathrm{pt}$ to test $\pm \mathbf{E}_{i}$ and select the lowest. Similarly, we allow for $\pm \mathbf{t}_{i}$ and the two rotation matrices induced by $\pm \mathbf{E}_{i}$ with $5 \mathrm{pt}$. In total, we generate 10,000 random instances of 3, 4 or 5 points using our data model, and we report on average performance.

Although $1+3$ pt only requires four locations in total, it is most vulnerable to noise, yet comparable to $5 \mathrm{pt}$ with similar results for t-error. $2+1 \mathrm{pt}$ performs significantly better than both other approaches, as the essential matrix derived is supported by two accurate scale measurements, and the solver is more stable.

\subsubsection{Noise in Scale}

We now have a closer look at $1+3 \mathrm{pt}$ and $2+1 \mathrm{pt}$ with noisy scale $(5 \mathrm{pt}$ is unaffected by scale). Fig. 4 shows the average performance for 10,000 instantiations of different noiselevels (modelled as Gamma distribution around 1 with a variance of one $100^{\text {th }}$ of SIFT's default step-size).

Again, $2+1$ pt out-performs the $1+3$ pt setup, and rotations are especially improved. Notice, in (11) two scales must ensure that the rotation supports the translation, reducing the chance of incorrect results. In fact, we found that for large errors in scale, $2+1 \mathrm{pt}$ often produce few or even no real valued solution, which indicates some robustness to noise.

Finally fig. 5 models noise in scale systematically with increasing error. Notice, E-error for both, $1+3 \mathrm{pt}$ and $2+1 \mathrm{pt}$, are virtually monotonously increasing with larger noise levels. This behaviour is essential for the bisection algorithm in section 4.2. We emphasize, although the true essential matrix is unknown in real data, an approximation of E-error is provided by estimating the inlier ratio, or alternatively the mean error in epipolar constraint. 

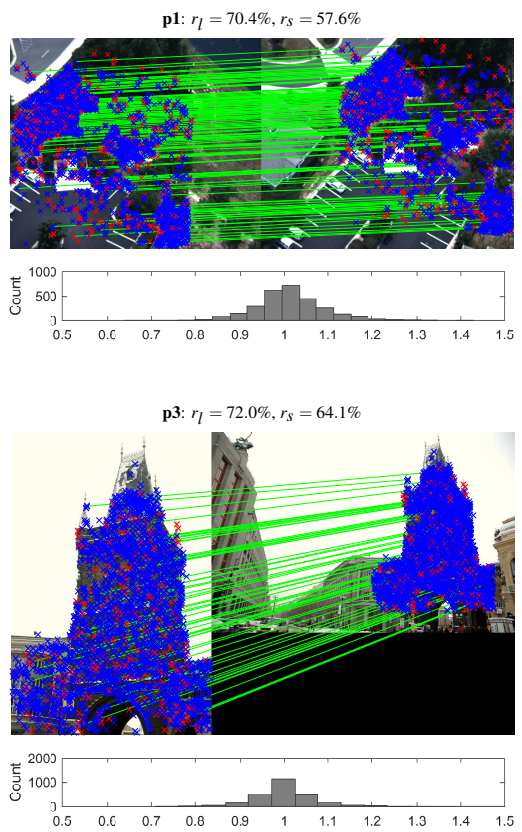

p5: $r_{l}=44.2 \%, r_{s}=38.4 \%$
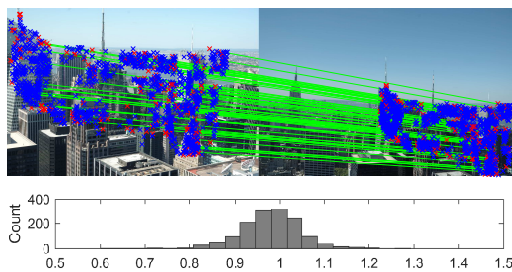

p2: $r_{l}=63.5 \%, r_{s}=52.5 \%$
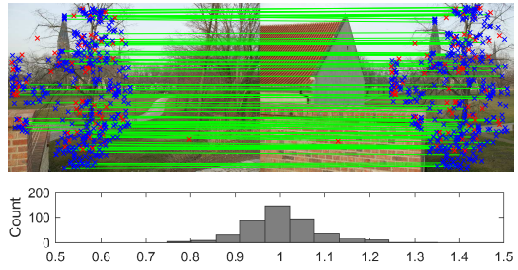

p4: $r_{l}=74.0 \%, r_{s}=68.8 \%$
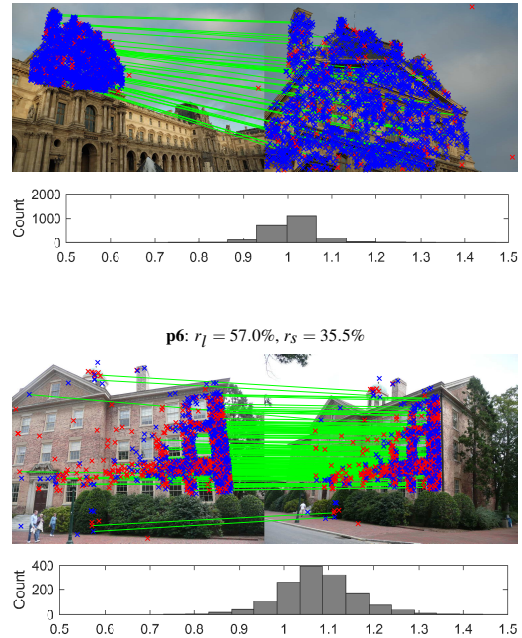

Figure 6: Location $(\times)$ and scale inlier $(\times)$ for $2+1^{*}$ on image pairs $\mathrm{p} 1$ to $\mathrm{p} 6$ are shown. The ratio of location inliers $r_{l}$ and scale inliers $r_{s}$ is listed, and a subset of feature correspondences is shown. The histogram of scale divergence $\frac{\hat{s}_{i}}{s_{i}}$ with true scale $\hat{s}_{i}$ as estimated by $2+1^{*}$ is shown under the image pairs.

\subsection{Real Data}

We test the proposed methods on 6 real image pairs with varying degree of difficulty (fig. 6). First we extract SIFT feature matches using the standard setup in [ㅁ]]. We then perform RANSAC (with $p=0.99, \delta_{l}=1 p x, \delta_{s}=0.1$ ), and our evaluation is based on the location inlier criterion. The comparison is between the proposed methods and the five point algorithm in [四]. As RANSAC is based on a random selection process, we instantiate all algorithms 1,000 times and report averages. Note, real data is effected by both, noise in scale and location.

We compare the inlier ratio for $5 \mathrm{pt}, 1+3 \mathrm{pt}$ and $2+1 \mathrm{pt}$ in fig. 7 . The performance without scale correction is significantly reduced for $1+3 \mathrm{pt}$ and $2+1 \mathrm{pt}-$ an indication that scale is much less reliable in real data than in our synthetic results. Fig. 7 additionally shows the results of $1+3 \mathrm{pt}$ and $2+1 \mathrm{pt}$ with bisection refinement (section 4.2 ), denoted $1+3^{*}$ and 


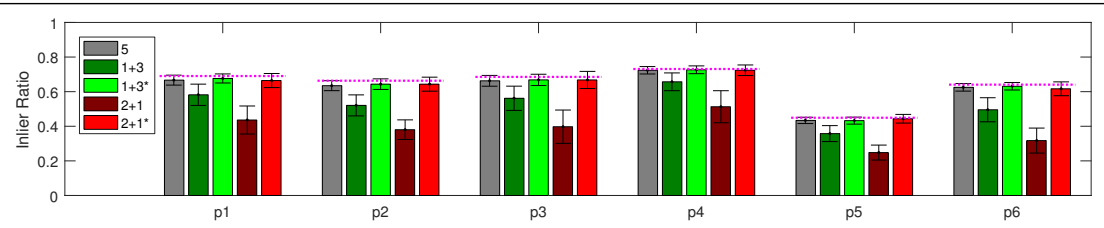

Figure 7: Location based inlier ratio for 1,000 iterations of RANSAC is shown with standard deviation. The magenta line represents performance of post-refined 5 point algorithm using non-linear optimization on all inlier points.

\begin{tabular}{|c|cccc|}
\hline Method & Iterations & Explored Solutions & Solver Runtime & Total Runtime \\
\hline $5 \mathrm{pt}$ & 83 & 373 & $162 \mathrm{~ms}$ & $32 \mathrm{~s}$ \\
$1+3^{*}$ & 49 & 1920 & $18 \mathrm{~ms}$ & $103 \mathrm{~s}$ \\
$2+1^{*}$ & 33 & 3810 & $6 \mathrm{~ms}$ & $201 \mathrm{~s}$ \\
\hline
\end{tabular}

Table 1: Average statistics of the different methods in RANSAC are listed using the naïve setting without early bailout. While the solver runtime for our methods is improved, the evaluation of the increased set of solutions (due to scale refinement) is costly in total runtime.

$2+1^{*}$. Overall these perform similarly to $5 \mathrm{pt}$, with $2+1^{*}$ reaching the highest inlier ratios in $\mathrm{p} 2, \mathrm{p} 3, \mathrm{p} 4$ and $\mathrm{p} 5$, and $1+3^{*}$ having the best performance in $\mathrm{p} 1$ and p6. Fig. 6 shows visual examples for $2+1^{*}$. Image pair $\mathrm{p} 1$ has limited scale change and relatively simple camera configuration. Images p2, p3, p4 and p5 benefit from scale as we obtain reduced location dependence. A challenging setting for scale exploitation is $\mathrm{p} 6$, as the projection reduces the area of the patches' ellipses consistently, resulting in incorrect scale ratios. This is also apparent in the divergence in scale, given by $\frac{\hat{s}_{i}}{s_{i}}$ with required scale $\hat{s}_{i}$ as estimated by $2+1^{*}$, which are normal distributions around 1 apart from results in p6 (fig. 6).

Tab. 1 shows detailed statistics of $1+3^{*}, 2+1^{*}$ and $5 \mathrm{pt}$ within the RANSAC setting. Overall $1+3^{*}$ and $2+1^{*}$ improve upon RANSAC iterations and the minimal solver is much faster than that of 5pt (using the MATLAB solvers generated by [ $[$ ] $]$ ). However, in our scale refinement we instantiate the solver $1+2^{3}=9$ times for $1+3^{*}$ and $1+(2+2)^{3}=65$ times for $2+1^{*}$ (with a greedy approach) leading to many explored solutions. Consequently, our proposed methods reduce in runtime. Here, early bailout [ $[\mathbf{}]$ and preemptive evaluation [ $\square$ ] is advantageous and essential with less reliable scales.

\section{Conclusion}

Exploiting intrinsically provided scale ratios from scale-space feature matching techniques, we have introduced two minimal solvers for the minimal sets of relative pose estimation from scale and location correspondences. We then integrated our framework to RANSAC, using a two class inlier set and proposed a bisection search for refinement of less reliable scale. Finally, we present a performance analysis with synthetic data and evaluate our approach in comparison with the five point algorithm in a RANSAC setting.

Overall, our $1+3$ and $2+1$ point algorithms produce comparable results, but with reduced dependence on location inliers. The presented solvers are magnitudes faster than that of [ㅁ] in our experiments. However, with unreliable scale ratios, scale refinement is necessary, and we introduce an efficient bisection search for this. Early bailout $[Q]$ and preemptive evaluation []] can further reduce runtime, and improved scale accuracy should be explored in future work. 


\section{References}

[1] O. Chum and J. Matas. Randomized RANSAC with $T_{d, d}$ Test. In $B M V C^{\prime} 02$, pages 448 $-457,2002$.

[2] B. Clipp, C. Zach, J. Frahm, and M. Pollefeys. A New Minimal Solution to the Relative Pose of a Calibrated Stereo Camera with Small Field of View Overlap. In IEEE Int. Conf. Computer Vision, ICCV'09, pages 1725-1732, 2009.

[3] A. Cohen and C. Zach. The Likelihood-Ratio Test and Efficient Robust Estimation. In IEEE Int. Conf. Computer Vision, ICCV'15, pages 2282 - 2290, 2015.

[4] W. Gellert, H. Küstner, M. Hellwich, and H. Kästner. The VNR Concise Encyclopedia of Mathematics. Springer, 1975.

[5] L. Goshen and I. Shimshoni. On Averaging Rotations. IEEE Trans. Pattern Anal. Mach. Intell., 30(7):1230 - 1242, Jul 2008.

[6] R. Hartley. In Defence of the Eight-Point Algorithm. IEEE Trans. Pattern Anal. Mach. Intell., 19(6):580-593, Jun 1997.

[7] R. Hartley and A. Zisserman. Multiple View Geometry in Computer Vision. Cambridge University Press, 2003.

[8] Z. Kukelova, M. Bujnak, and T. Pajdla. Automatic Generator of Minimal Problem Solvers. In ECCV'08, pages 302-315, 2008.

[9] G. Lee, F. Fraundorfer, and M. Polleyfeys. Motion Estimation for Self-Driving Cars with a Generalized Camera. In CVPR'13, 2013.

[10] B. Li, L. Heng, G. Lee, and M. Polleyfeys. A 4-Point Algorithm for Relative Pose Estimation of a Calibrated Camera with a Known Relative Rotation Angle. In IROS'13, pages 1595-1601, 2013.

[11] H. Li. A Simple Solution to the Six-Point Two-View Focal-Length Problem. In ECCV'06, pages 200-213, 2006.

[12] D. Lowe. Distinctive Image Features from Scale-Invariant Keypoints. Int. J. Comput. Vis., 60(2):91 - 110, 2004.

[13] Perdoch M., J. Matas, and Chum O. Epipolar Geometry from Two Correspondences. In Int. Conf. Pattern Recognition, ICPR'06, pages 215-219, 2006.

[14] O. Naroditsky, X. Zhou, J. Gallier, S. Roumeliotis, and K. Daniilidis. Two Efficient Solutions for Visual Odometry using Directional Correspondence. IEEE Trans. Pattern Anal. Mach. Intell., 34(4):818-824, Apr 2012.

[15] D. Nistér. Preemptive RANSAC for Live Structure and Motion Estimation. In IEEE Int. Conf. Computer Vision, ICCV'03, pages 199-206, 2003.

[16] D. Nistér. An Efficient Solution to the Five-Point Relative Pose Problem. IEEE Trans. Pattern Anal. Mach. Intell., 26(6):756-770, Jun 2004. 
[17] C. Raposo and J. Barreto. Theory and Practice of Structure-From-Motion Using Affine Correspondences. In CVPR'16, pages 5470 - 5478, 2016.

[18] E. Rublee, V. Rabaud, K. Konolige, and G. Bradski. ORB: an efficient alternative to SIFT or SURF. In IEEE Int. Conf. Computer Vision, ICCV'11, pages 2564-2571, 2011.

[19] Scaramuzza. 1-Point-RANSAC Structure from Motion for Vehicle Mounted Cameras by Exploiting non-Holonomic Constraints. Int. J. Comput. Vis., 95(1):74 - 85, Jan 2011.

[20] H. Stewénius, D. Nistér, F. Kahl, and F. Schaffalitzky. A Minimal Solution for Relative Pose with Unknown Focal Length. Image and Vision Computing, 26(7):871 - 877, Jul 2008. 